\title{
Homozygosity mapping of spinocerebellar ataxia with cerebellar atrophy and peripheral neuropathy to 9q33-34, and with hearing impairment and optic atrophy to 6p21-23
}

\author{
Pascale Bomont ${ }^{1}$, Mitsunory Watanabe ${ }^{2}$, Ruth Gershoni-Barush ${ }^{3}$, Masami Shizuka ${ }^{2}$, \\ Makoto Tanaka ${ }^{2}$, Jinpei Sugano ${ }^{4}$, Christophe Guiraud-Chaumeil ${ }^{1}$ and Michel Koenig ${ }^{1}$ \\ ${ }^{1}$ Institut de Génétique et de Biologie Moléculaire et Cellulaire, CN RS/IN SERM/Université Louis-Pasteur, IIlkirch, \\ France; ${ }^{2}$ Department of Neurology, Gunma University School of Medicine, Maebashi, Japan; ${ }^{3}$ Rambam Medical \\ Center, Technion Israel Institute of Technology, Haifa, Israel; ${ }^{4}$ Social Insurance Gunma Chuo General Hospital \\ Neuromedecine, Maebashi, Japan
}

With the availability of a simple molecular test that distinguishes Friedreich ataxia, the most frequent form of inherited ataxia, from other recessive ataxias, it now becomes possible to unravel the genetic heterogeneity of the latter. We have now localised two genes causing autosomal recessive spinocerebellar ataxia in two consanguineous families. In the first family, the four affected Japanese sibs had spinocerebellar ataxia associated with elevated levels of serum creatine kinase, $\gamma$-globulin, and $\alpha$-foetoprotein. Homozygosity over a $20 \mathrm{cM}$ region allowed to demonstrate linkage at 9q33.3-34.3 with a lod score of 3.0. Genotyping two unrelated Japanese patients from first degree consanguineous parents revealed that one was homozygous for the same region but did not share the biochemical features. In the second family, an Israeli uncle and a niece were affected by an early-onset recessive ataxia and subsequently developed hearing impairment and optic atrophy. Homozygosity over a $17 \mathrm{cM}$ region allowed demonstration of linkage at 6p21-23 with a lod score of 3.25. These two localisations of autosomal recessive ataxia genes represent a first step toward the identification of genetically homogenous, non-Friedreich, ataxic patients and subsequent cloning of the genes. European Journal of Human Genetics (2000) 8, 986-990.

Keywords: ataxia; cerebellar; $\alpha$-foetoprotein; immunoglobulin; neuropathy; recessive; homozygosity; mapping

\begin{abstract}
Introduction
Autosomal recessive ataxias are neurodegenerative disorders that affect the cerebellum and/or the spinocerebellar tracts and the posterior columns of the spinal cord. The major forms of recessive ataxia can often be distinguished on clinical grounds. Friedreich ataxia (FRDA) usually associate limb and gait ataxia, cerebellar dysarthria, extensor plantar reflexes, and reduced or absent tendon reflexes, loss of vibration/position sense and muscle weakness in lower limbs with hypertrophic cardiomyopathy, scoliosis, pes cavus and an increased frequency of impaired glucose tolerance and
\end{abstract}

Correspondence: Michel Koenig, Institut de Génétique et de Biologie Moléculaire et Cellulaire, 1 rue Laurent Fries BP163, 67404 Illkirch cedex, CU de Strasbourg, France. Tel: +33 38865 3399; Fax: + 33388653246 ; E-mail: mkoenig@igbmc.u-strasbg.fr

Received 31 July 2000; revised 24 October 2000; accepted 24 October 2000 diabetes. ${ }^{1,2}$ Ataxia-telangiectasia ${ }^{3}$ is associated with conjunctival telangiectasias, immunodeficiency and an increased incidence of lymphoma and leukaemia. Serum gammaglobulins are reduced, and alpha-foetoprotein and carcinoembryonic antigen are elevated. Rarer forms of recessive ataxias have been recognised, often due to clustering, by founder events, of patients in precise geographic areas. Ataxia with isolated vitamin E deficiency (AVED), frequent in North Africa, overlaps clinically with FRDA despite a lower frequency of cardiomyopathy and is easily diagnosed by serum vitamin E measurements. ${ }^{4}$ Spastic ataxia of CharlevoixSaguenay (ARSACS), frequent in the Charlevoix-Saguenay region of Québec, presents with spasticity and brisk tendon reflexes. ${ }^{5}$ Infantile on set spinocerebellar ataxia (IOSCA) has been so far described only in Finland and can be distinguished by a significant reduction in sensory nerve conduction velocities. ${ }^{6}$ 
Major breakthroughs have been achieved for the understanding of Friedreich ataxia and ataxia-telangiectasia through the cloning of their defective genes. ${ }^{7,8}$ The cloning or localisation of the genes involved in AVED, ARSACS and IOSCA has also been achieved in recent years, thanks to linkage disequilibrium studies of the founder events in the populations involved. ${ }^{9-11}$ Friedreich ataxia-like patients with normal vitamin E levels are likely to represent a heterogeneous group of diseases with little possibility to define a nosological classification for patients from outbred populations. We show here that consanguineous families with at least two affected were useful for the localisation of two new genes, on 9q33.3-34.3.3 and 6p21-23, as a first step toward the unravelling of the genetic complexity of this condition.

\section{Subjects and methods Subjects}

The presentation of the four patients of family 1 , from Japan, is described in detail elsewhere. ${ }^{12}$ Briefly, onset was around 20 years in all four cases; the patients, born of first-degree consanguineous parents, had spinocerebellar ataxia with cerebellar atrophy, telangiectasia over the bulbar conjunctiva and elevated $\alpha$-foetoprotein. However, $\gamma$-globulins were also elevated and the diagnosis of a mild form of ataxiatelangiectasia was excluded by linkage studies. Friedreich ataxia was excluded both by linkage and absence of GAA expansion in the frataxin gene. The patients also presented peripheral sensory neuropathy, nystagmus, saccadic eye movements but no ocular motor apraxia. Creatine kinase was moderately elevated, vitamin E level was normal, as well as all other biological tests. ${ }^{12}$ The father is deceased and samples of the healthy sibs were not available for study.

Two other Japanese patients with Friedreich ataxia-like presentation (families 2 and 3) and born of first-degree consanguineous parents were included later in the study. The patient from family 2 had onset of symptoms at 22 years of age, became wheelchair-bound at age 26 and is now 74 years old. She has marked upper-limb ataxia, ataxic dysarthria, nystagmus, saccadic eye movements, head tremor, deformed hands in the flexed position and predominant proximal muscle atrophy. She has no areflexia, no telangiectasia, no hearing loss and no urinary disturbances. She had a younger brother who died of the same disease (no DNA available for study). The patient from family 3 had onset of symptoms at 52 years of age. He developed gait, and upper and lower limbs ataxia, dysarthria, lower limbs areflexia, moderately disturbed vibration sense, bilateral impaired hearing and diabetes mellitus. Families 1, 2 and 3 were not related to each other. The patients from families 2 and 3 had normal levels of $\alpha$-foetoprotein, $\gamma$-globulins, creatine kinase and vitamin $E$, and no GAA expansion.

In family 4, from Israel, an uncle and a niece were affected by an early onset Friedreich-like ataxia. The uncle started with gait ataxia at age 3 and is now wheelchair-bound and almost deaf at the age of 27 . He has nystagmus, visual decline, and hand contractures. Fundus examination revealed bilateral optic atrophy. The niece also started with gait ataxia at 3 years of age. Examination at age 6 revealed no cranial nerve involvement. Blood tests included aldolase, CPK, LDH, vitaminsE and B12, folic acid, creatinine, glucose, free fatty acids, ketones, lactate and pyruvate; all were normal. At the same time, a muscle biopsy was performed that revealed mild fibre disproportion but no evidence of mitochondrial disease. At age 14, she showed slow responses on electroretinograms. She is now 17 years old and has impaired hearing and vision but no nystagmus. The diagnosis of Friedreich ataxia was excluded by linkage analysis and absence of GAA expansion. The parents of both affected individuals are related by two intricated first-degree, onceremoved consanguinity loops (Figure1). The parents and two healthy sibs of the affected niece were also included in whole genome typing.

Two consanguineous Lebanese families with Friedreich ataxia-like presentation but without GAA expansion were included later in the study. In one family, two children, born of first-degree consanguineous parents, presented with early onset ataxia plus sensorimotor neuropathy. In the other family, the two patients were born of second-degree consanguineous parents.

Blood samples were obtained with informed consent. Genomic DNA was extracted from the peripheral blood leukocytes by a standard phenol/chloroform method. Samples representing 40 Japanese and 20 Israeli/Lebanese independent chromosomes were analysed for estimation of allele frequency.

\section{Genotyping}

Genomewide scan was performed with the ABI PRISM Linkage Mapping Set Version 2 (PE Applied Biosystems, Foster City, CA, USA). This screening set comprises 380 polymorphic fluorescently-labelled microsatellite markers (CA/TG), with an average spacing of $10 \mathrm{cM}$ between markers. PCR reactions were done in a $10 \mu \mathrm{l}$ final volume with dilutions as recommended by the manufacturer. Pooled PCR products were analysed on an ABI 377DNA sequencer (PE Applied Biosystems). The data were analysed using $A B I$ PRISM GeneScan Analysis Software and Genotyper Software. After suggestion for linkage was obtained, additional CA/TG microsatellite markers ${ }^{13}$ from the candidate regions were tested. Genotypes were determined by PCR amplification using one of the primers labelled with ${ }^{32} \mathrm{P}$. Annealing temperature for PCR was $2^{\circ} \mathrm{C}$ below the lowest Tm of the two primers. The PCR products were separated on $6 \%$ denaturing polyacrylamide gels and visualised by autoradiography.

\section{Linkage analysis}

Part of the linkage power of the families resides in the consanguinity loop(s) and linkage is supported when the patients are homozygous for a rare haplotype. This information was included in two-point lod score calculation by considering the non-recombinant haplotype as a single 
locus. ${ }^{14}$ The frequency of the homozygous haplotype was calculated as the product of the frequency of the individual alleles estimated from the reference population in the relevant countries. In order to eliminate bias due to possible linkage disequilibrium, only one marker was taken into account when two were located at the same position on the genetic map. Two-point lod scores with consanguinity loops were calculated by using the MLINK program of the LINKAGE package. ${ }^{15}$ We assumed a fully penetrant autosomal recessive mode of inheritance, and a gene frequency of 0.005 which certainly represents an upper limit for these rare conditions.

\section{Results}

The entire autosomal set of the ABI PRISM Linkage Mapping Set was tested on families 1 and 4 . Given the close consanguinity of the parents and the close spacing of the

Family

\#1

\#2

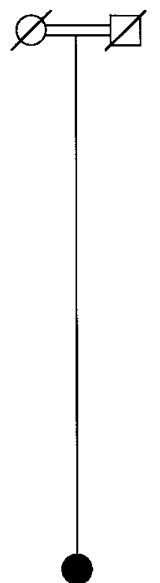

CM $\underline{\$ 1776}$

$9 \underline{\$ 1682}$

$9 \lcm{\$ 290}$

$2 \quad \$ 159$

$1 \quad S 1831$

151863

1 S1847

0 $\quad \$ 179$

$1 \quad \$ 1830$

$3 \quad \$ 1793$

- $\underline{\$ 164}$

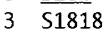

2 S114

$7 \quad \$ 1826$

$3 \quad \underline{5158}$

$4 \quad 51838$

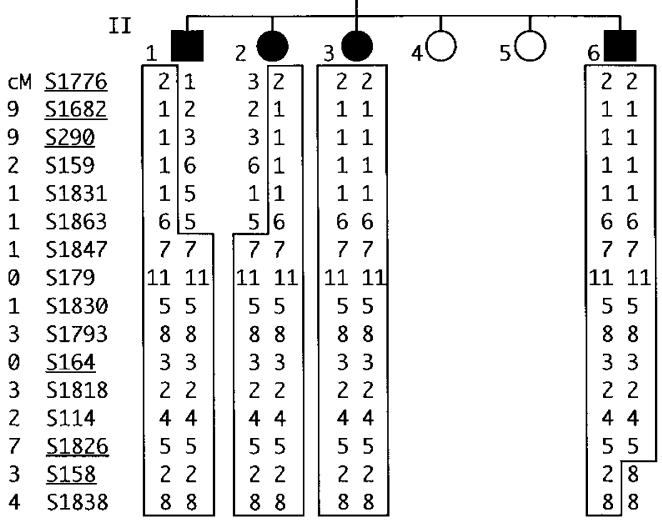

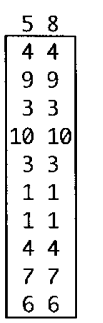

markers, we assumed that at least one marker from the linked region would be homozygous in all affected individuals in a given family. We identified 31 markers in family 1 and 19 markers in family 4 that appeared non-recombinant and homozygous in the affected (in a few cases markers were consecutive on the map). For these markers, haplotypes were constructed with one or two distal and proximal markers (three to five markers haplotype) depending on the informativeness of the haplotypes, allowing most regions to be excluded from linkage.

In family 1, only two regions (each comprising two consecutive markers) appeared informative and non-recombinant: one around D3S1266-D3S1277 and another around D9S164-D9S1826. The study of a dense set of microsatellite markers from the two regions ( $\mathrm{Dib}$ et $\mathrm{al}^{13}$ ) reveal ed that the chromosome 3 region was indeed homozygous by descent in

\#3

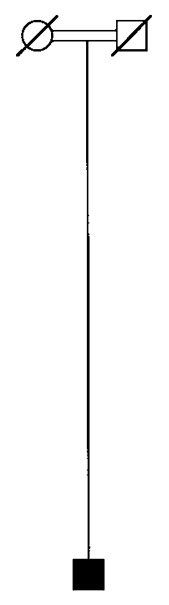

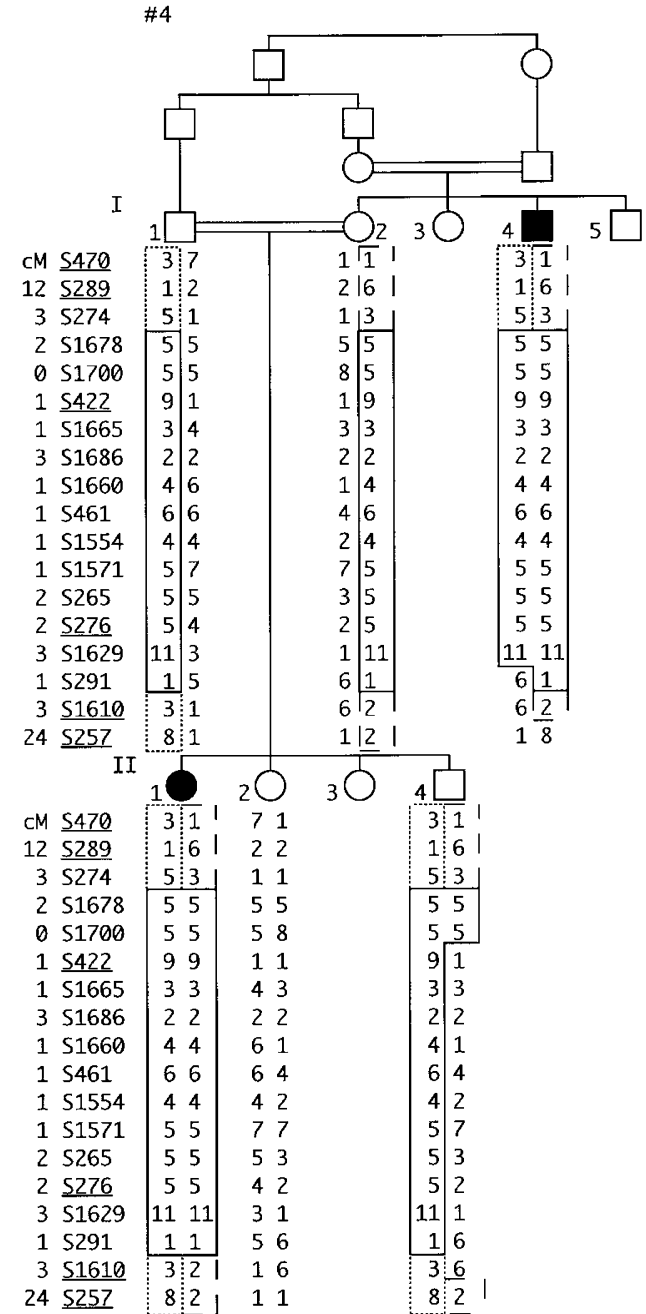

Figure 1 Allele segregation of linked and flanking markers in the autosomal recessive ataxia families. Markers in the pter-qter ordering are shown from top to bottom. The linked haplotypes are boxed. In family 1 , haplotypes of the father were deduced and are shown in italics. In family 4, the paternal and maternal haplotypes that are not homozygous in the patients are boxed with dotted and dashed lines, respectively. Distance (in CM) from the previous (above) marker is indicated in front of each marker. Markers that were part of the whole genome initial typing are underlined; nt: not tested 
three affected sibs (homozygosity over 12 consecutive markers) but was entirely excluded in the fourth sib (not shown), whilst the chromosome 9 region appeared homozygous by descent and non-recombinant for all patients (Figure1). The highest lod score (2.65) was obtained with marker D9S1830 at $\theta=0$ (Table1). Two-point lod score calculation with the non-recombinant haplotype gave a value of $Z=+3.00$ at $\theta=0$. The recombinant markers D9S1863 (centromeric) and D9S158 (distal) define a $20 \mathrm{cM}$ interval located in $9 q 33.3-34.3 .3$. Patients $I 13$ and $\| 6$ are homozygous over a larger region (over 46 and $39 \mathrm{cM}$, respectively), strengthening homozygosity by descent.

In family 4, there was only one region in which the two patients were homozygous for two consecutive and informative markers (D6S422 and D6S276), and the affected uncle and his related brother-in-law shared a disease haplotype over four consecutive markers (the two previous markers plus D6S470 and D6S289, Figure1). The study of additional markers from this region ${ }^{13}$ revealed that the two patients were indeed homozygous by descent (homozygosity over 12 consecutive markers, Figure 1 ). The highest lod score (2.77) was obtained with marker D6S1660 at $\theta=0$ (Table 1 ). Two-point lod score calculation with the non-recombinant hapl otype gave a value of $Z=+3.25$ at $\theta=0$. The recombinant markers D6S291 (centromeric) and D6S1700 (distal) define a $17 \mathrm{cM}$ interval located in 6p21-23.

In order to search for additional families linked to either of the two loci, we genotyped two independent Japanese ataxia patients born of first-degree consanguineous parents and two consanguineous Lebanese families. All these patients had initially a diagnosis of Friedreich-like ataxia that was subsequently ruled out by linkage and lack of expansion mutation.

Table 1 Lod score at $\theta=0$ for each individual marker and for the linked haplotype in families 1 and 4

\begin{tabular}{|c|c|c|c|c|c|}
\hline & nily 1 & & & amily 4 & \\
\hline $\begin{array}{l}\text { Marker } \\
\text { or } \\
\text { haplotype }\end{array}$ & $\begin{array}{l}\text { Frequency } \\
\text { of linked } \\
\text { allele }\end{array}$ & $\begin{array}{l}\text { Lod } \\
\text { score }\end{array}$ & $\begin{array}{l}\text { Marker } \\
\text { or } \\
\text { haplotype }\end{array}$ & $\begin{array}{l}\text { Frequency } \\
\text { of linked } \\
\text { allele }\end{array}$ & $\begin{array}{l}\text { Lod } \\
\text { score }\end{array}$ \\
\hline D9S1863 & 0.33 & $-\infty$ & D6S274 & 0.5 & $-\infty$ \\
\hline D9S1847 & 0.05 & 2.58 & D6S1700 & 0.5 & 0.92 \\
\hline D9S179 & 0.08 & 2.42 & D6S422 & 0.45 & 1.33 \\
\hline D9S1830 & 0.04 & 2.65 & D6S1665 & 0.18 & 0.93 \\
\hline D9S1793 & 0.10 & 2.34 & D6S1686 & 0.5 & 0.43 \\
\hline D9S164 & 0.3 & 0.70 & D6S1660 & $\leqslant 0.05$ & 2.77 \\
\hline D9S1818 & 0.11 & 2.30 & D6S461 & 0.6 & 1.07 \\
\hline D9S114 & 0.33 & 0.70 & D6S1554 & 0.4 & 1.40 \\
\hline D9S1826 & 0.26 & 1.86 & D6S1571 & $\leqslant 0.05$ & 2.75 \\
\hline D9S158 & 0.33 & $-\infty$ & D6S265 & 0.5 & 1.22 \\
\hline & & & D6S276 & 0.13 & 2.30 \\
\hline & & & D6S1629 & 0.14 & 2.26 \\
\hline & & & D6S291 & 0.45 & -1.56 \\
\hline D9S1847- & & & D6S422- & & \\
\hline $\begin{array}{l}\text { D91826 } \\
\text { haplotype }^{a}\end{array}$ & $2 \times 10^{-6}$ & 3.00 & $\begin{array}{l}\text { D6S1629 } \\
\text { haplotype }\end{array}$ & $2.2 \times 10^{-7}$ & 3.25 \\
\hline
\end{tabular}

amarkers D9S179 and D9S164 were not included in the haplotype, due to possible linkage disequilibrium with markers D9S1847 and D9S1793, respectively.
One Japanese patient (family 2 ) showed homozygosity over the region of linkage of family 1 (Figure 1), whilst the other families were linked at none of the two loci. The patient from family 2 had a similar age of onset and clinical evolution as patients from family 1 but had none of the biological markers found in family 1 (elevated serum creatine kinase, $\gamma$-globulin, and $\alpha$-foetoprotein). Despite the fact that the patients from the other families had additional clinical features, it is not possible to lump families 1 and 2 on clinical grounds alone and the lod score of the two families could not be combined.

\section{Discussion}

Recessive ataxias without significant cognitive impairment represent a heterogeneous group of neurodegenerative diseases with important clinical variability and phenotypic overlap. With the exception of AT and AVED, where diagnosis is confirmed, respectively, by elevated $\alpha$-foetoprotein and very low serum vitamin $E$, there is no specific biological marker to help to distinguish the other forms of recessive ataxia, and diagnosis remains based on clinical presentation and disease progression. The finding of a GAA triplet repeat expansion on at least one chromosome9 (most often on both) in FRDA patients has modified the diagnostic procedure of this frequent cause of inherited ataxia. ${ }^{7}$ Only $38 \%$ of children/adolescents and $11 \%$ of adults with recessive ataxia have a confirmed molecular diagnosis of FRDA, and proportions are even smaller for sporadic cases, leaving ample space for genetic heterogen eity. ${ }^{16,17}$ The recent cloning of the gene involved in ARSACS, ${ }^{10}$ encoding a new protein of unknown function, may facilitate the diagnosis of this rare condition, by a western blot approach, as is already the case for autosomal recessive muscular dystrophies. Several genes disrupted in recessive ataxias point to a pathological mechanism involving oxidative stress. This is the case for AVED, by severe reduction of serum vitamin $E$ which is the most potent membrane anti-oxidant, but also for FRDA and X-linked sideroblastic anaemia and ataxia (XLSA/A) where disturbance of iron or iron-sul phur cluster homeostasis are likely to cause oxidative stress by Fenton chemistry. ${ }^{18}$ Similarly, the cerebellar symptoms seen in AT may be accounted for by an improper response against free radicals produced by ionising radiations. ${ }^{19}$ Theidentification of genes involved in the other forms of recessive ataxia should help to design new diagnostic tools and gain a better understanding of the underlying pathophysiology (and hence better strategies for therapeutic intervention).

We show here that isolated consanguineous families can be extremely useful in defining new loci of autosomal recessive ataxia. In particular, linkage in family 4 could be identified only because the two patients from this family descend from two partially independent consanguineous marriages. Homozygosity by descent of the two patients is sufficient to obtain a lod score of 3.0 in favour of linkage with the homozygous haplotype, the study of the two healthy sibs giving little 
additional contribution to the final lod score. The study of the healthy sibs was very useful however during the genomewide survey for exclusion of many loci.

The next step will consist of identification of additional families linked to either one of these two loci. Our initial attempt to do so was only moderately successful, since only one patient from an independent family could possibly be linked to the 9q33.3-34.3 region. Given first degree consanguinity of the parents, this would yield a lod score of 1.2 in favour of linkage at this locus. However, given the fact that multiple tests for linkage were performed in consanguineous families, we cannot rule out the possibility that the patient from family 2 is homozygous for the 9q33.3-34.3 haplotype only by consanguinity and not by linkage. The identification of additional families linked to either one of these two loci can initially only be performed on large or informative families. Linkage can be demonstrated if the posterior probability of linkage in a given family is at least 95\%, corresponding to a lod score of $2.56 .^{20}$ The finding of additionally linked families would be invaluable in identifying clinical or biological parameters that are characteristic of one or the other locus and would in turn allow analysis of the smaller families.

Many defective functions could in principle lead to a neurodegenerative phenotype with ataxia as a prominent feature. However, no obvious candidate gene was present in the candidate intervals. The gene encoding the bullous pemphigoid antigen 1 (BPAG1) protein (also called actinbinding protein 280 or ABP280) has an alternative form specifically expressed in dorsal root ganglia, causes sensory ataxia when altered in the mouse and is local ised on human $6 p 11-12 .^{21}$ It is however located between the markers D6S1636 and D6S257 (on radiation hybrid cell lines), a region centromeric to $\mathrm{D} 6 \mathrm{~S} 29 \mathrm{I}^{22}$ and is therefore excluded from the candidate interval of family 4 . Extensive human genome sequencing efforts should help to identify new markers and new candidate genes that will enable identification of mutations in rare disorders, once a genetic localisation is defined. We have shown here that isolated families with an autosomal recessive disease can contribute significantly to this work.

\section{Acknowledgements}

We wish to thank Professor JL Mandel for support and helpful discussions, Dr. André Mégarbane for referring DNA samples of the Lebanese families, S Vicaire, N Lagarde, I Colas, F Ruffenach and E Troesch for excellent technical help. This work was supported by funds from the Institut National de la Recherche Médicale (INSERM), the Centre National de la Recherche Scientifique (CNRS), the Hôpital Universitaire de Strasbourg (HUS), the Human Frontier Science Program (HFSP) and the Association Française contre les Myopathies (AFM) (to MK), and by a fellowship from the Association Française de I'Ataxie de Friedreich (AFAF) (to PB).

\section{References}

1 Harding AE: Friedreich's ataxia: a clinical and genetic study of 90 families with an analysis of early diagnostic criteria and intrafamilial clustering of clinical features. Brain 1981; 104: 589-620.

2 Durr A, Cossee M, Agid Y et al: Clinical and genetic abnormalities in patients with Friedreich's ataxia. N Engl J Med 1996; 335: 1169-1175.

3 Jeret JS, Lechtenberg R: Ataxia-telangiectasia. In: Lechtenberg R (ed). Handbook of Cerebeller Diseases. Marcel Dekker: New York, 1993, pp 477-490.

4 Cavalier L, Ouahchi K, Kayden HJ et al: Ataxia with isolated vitamin E deficiency: heterogeneity of mutations and phenotypic variability in a large number of families. Am J Hum Genet 1998; 62: 301-310.

5 Bouchard JP, Richter A, Mathieu J et al: Autosomal recessive spastic ataxia of Charlevoix-Saguenay. Neuromusc Disord 1998; 8: 474-479.

6 Koskinen T, Santavuori P, Sainio K, Lappi M, Kallio AK, Pihko H: Infantile onset spinocerebellar ataxia with sensory neuropathy: a new inherited disease. J Neurol Sci 1994; 121: 50-56.

7 Campuzano V, Montermini L, Molto MD et al: Friedreich's ataxia: autosomal recessive disease caused by an intronic GAA triplet repeat expansion. Science 1996; 271: 1423-1427.

8 Savitsky K, Bar-Shira A, Gilad S et al: A single ataxia telangiectasia gene with a product similar to PI-3 kinase. Science 1995; 268: 1749-1753.

9 Ouahchi K, Arita M, Kayden $\mathrm{H}$ et al: Ataxia with isolated vitamin $\mathrm{E}$ deficiency is caused by mutations in the alpha-tocopherol transfer protein. Nat Genet 1995; 9: 141-145.

10 Engert JC, Berube P, Mercier J et al: ARSACS, a spastic ataxia common in northeastern Quebec, is caused by mutations in a new gene encoding an 11.5-kb ORF. N at Genet 2000; 24: 120-125.

11 Nikali K, Isosomppi J, Lonnqvist T, Mao JI, Suomalainen A, Peltonen L: Toward cloning of a novel ataxia gene: refined assignment and physical map of the IOSCA locus (SCA8) on 10q24. Genomics 1997; 39: 185-191.

12 Watanabe M, Sugai $Y$, Concannon $P$ et al: Familial spinocerebellar ataxia with cerebellar atrophy, peripheral neuropathy, and elevated level of serum creatine kinase, gamma-globulin, and al phafetoprotein. Ann Neurol 1998; 44: 265-269.

13 Dib C, Fauré S, Fizames C et al: A comprehensive genetic map of the human genome based on 5,264 microsatellites. Nature 1996; 380: 152-154.

14 Ben Hamida C, Doerflinger N, Belal S et al: Localization of Friedreich ataxia phenotype with selective vitamin E deficiency to chromosome $8 q$ by homozygosity mapping. Nat Genet 1993; 5: 195-200.

15 Lathrop GM, Lalouel JM, Julier C, Ott J: Multilocus linkage analysis in humans: detection of linkage and estimation of recombination. Am J Hum Genet 1985; 37: 482-498.

16 Moseley ML, Benzow KA, Schut LJ et al: Incidence of dominant spinocerebellar and Friedreich triplet repeats among 361 ataxia families. Neurology 1998; 51: 1666-1671.

17 Gunaratne PH, Richards CS: Estimated contribution of known ataxia genes in ataxia patients undergoing DNA testing. Genet Test 1998; 1: 275-278.

18 Puccio $\mathrm{H}$, Koenig $\mathrm{M}$ : Recent advances in the molecular pathogenesis of Friedreich ataxia. Hum Mol Genet 2000; 9: 887-892.

19 Rotman G, Shiloh Y: Ataxia-telangiectasia: is ATM a sensor of oxidative damage and stress? Bioessays 1997; 19: 911-917.

20 Ott J (ed): Analysis of Human Genetic Linkage. Johns Hopkins University Press: Baltimore, 1991, pp 207-208.

21 Brown A, Bernier G, Mathieu M, Rossant J, Kothary R: The mouse dystonia musculorum gene is a neural isoform of bullous pemphigoid antigen 1. Nat Genet 1995; 10: 301-306.

22 Deloukas P, Schuler GD, Gyapay G et al: A physical map of 30,000 human genes. Science 1998; 282: 744-746. 